\title{
Diastema medio interincisal y su relación con el frenillo labial superior: una revisión
}

\section{María Elena Díaz Pizán}

Docente del Departamento Académico de Estomatología del Niño y del Adolescente. Facultad de Estomatología. Universidad Peruana Cayetano Heredia.
Díaz ME. Diastema medio interincisal y su relación con el frenillo labial superior: una revisión. Rev Estomatol Herediana 2004;14(1-2) : 95 - 100.

\section{RESUMEN}

El diastema medio interincisal es una característica normal en la dentición decidua y mixta, la cual tiende a desaparecer posteriormente con la erupción de los caninos y segundas molares superiores permanentes. La mayoría de investigadores coinciden en señalar que el frenillo labial superior anómalo puede ser un importante factor etiológico en casos de diastemas patológicos, existiendo también otros factores como: hábitos perniciosos prolongados, desequilibrio muscular de la cavidad bucal, impedimento físico, anomalías dentales, maloclusiones etc. Un examen minucioso nos llevará a un diagnostico preciso a fin de establecer la conducta mas adecuada en el momento oportuno para el niño.

Palabras Clave: DIASTEMA / FRENILLO LABIAL / DENTICIÓN PRIMARIA.

Interincisal mid diasthema and its relation with the upper lip frenum: a review ABSTRACT

Interincisal mid diasthema is a normal characteristic in primary and mixed dentition which tends to dissapear with eruption of maxillary cuspids and second permanent molars. Most researchers agree that an abnormal upper lip frenum might be an important ethiological factor in pathologic diasthemas, besides other factors such as prolongued pernicious habits, muscular disequilibrium of the oal cavity, physical disability, dental anomalies, malocclusions, etc. A thorough examination will lead us to a precise diagnosis in order to establish the adequate treatment for the child.

Keywords: DIASTEMA / LABIAL FRENUM / PRIMARY DENTITION.
A lo largo de los años el Diastema Medio Interincisal (DMI) le han dado diferentes denominaciones, tales como "espacio de los incisivos centrales superiores” (1), “espacio de la línea media” (2,3), “diastema maxilar” (4), “diastema maxilar central” $(5,6)$, “diastema maxilar de la línea media” (7), “diastema maxilar interincisal” (8), “diastema medio o mediano” (9-13), “diastema mediano superior” (14), “diastema de la línea media” (15-20), “diastema anterior” (21), “diastema incisal”, (22,23) “diastema interincisivo o interincisal” $(24,25)$, “diastema mediano interincisal” (26), “diastema maxilar de la línea media” (27-29) o simplemente “diastema” (30-31).

$\mathrm{Al}$ diastema se le define como el espacio que existe entre los incisivos centrales superiores (16). Es una característica normal en la dentición decidua y mixta (32), que tiende a cerrarse luego de la erupción de los caninos perma- nentes y segundas molares, como resultado de la erupción, migración y ajuste fisiológico de las piezas dentarias $(9,15,16)$.

En la infancia, los incisivos centrales deciduos erupcionan casi inevitablemente con un espacio entre ellos $(2,8,33,34)$, el cual tiende a reducirse con la erupción de las otras piezas dentarias (2).

En 1950, Baume (32) contribuyó a aclarar el concepto de que los espacios en la dentición decidua son congénitos y no producto del desarrollo. Luego de evaluar modelos seriados de niños durante el periodo de erupción de los incisivos permanentes, concluyó que en arcos con incisivos deciduos espaciados (Tipo I), por lo general erupcionarían dientes anteriores adecuadamente alineados; mientras que en arcos con incisivos deciduos sin espacios (Tipo II), aproximadamente el $40 \%$ podían presentar apiñamiento anterior.
Por otro lado, los gérmenes de los incisivos centrales permanentes se desarrollan en criptas independientes, dentro del hueso, separados por el septum de la maxila $(35,36)$. Según Gibbs (1968) (37) “son los únicos dientes que erupcionan en criptas separadas por una sutura ósea bien definida” y posteriormente erupcionan de manera peculiar, con un espacio transitorio y muy acentuado entre ellos. A este estadio Broadbent (1941) (38) llama "etapa de patito feo", y es descrito como parte del proceso normal de desarrollo de la dentición permanente.

Todos estos acontecimientos favorecerían, en algunos casos, la presencia de un frenillo labial grande, el cual persistiría hasta que los incisivos entren en contacto proximal (36). Con la erupción, primero de los incisivos laterales y luego de los caninos permanentes, se inicia la “atrofia por presión” del frenillo (35). Esos dientes emergentes ejercerían 
una acción tipo cuña que, sumada al crecimiento del proceso alveolar, llevaría a la migración apical de la inserción gingival (9,35,39,40), y luego la erupción de las segundas molares permanentes superiores permitiría los ajustes necesarios para el cierre del espacio dental de la línea media (9).

Los estudios longitudinales de Bergström et al. (1973) (19), Popovich et al.(1977) (8) y transversales de Taylor (4) en 1939 y Weyman (13) en 1967 confirman la hipótesis de que el diastema maxilar de la línea media va disminuyendo a medida que aumenta la edad del individuo, siendo las épocas de mayor prevalencia de este espacio, antes de la erupción de los incisivos laterales.

Bergström et al. (19) en 1973 iniciaron el seguimiento de dos grupos, de 20 niños cada uno, con una edad promedio de 8 a 9 años, durante un período de 10 años, con el objetivo de determinar el curso del cierre del diastema y su relación con el Frenillo Labial Superior (FLS). A uno de los grupos se le practicó la frenectomía y al otro no. Al cabo de dos años la evaluación demostró que el diastema era considerablemente menor en los niños con frenectomía. Y luego de cinco años, las diferencias continuaban siendo significativas entre ambos grupos. Diez años después, los resultados mostraron que las diferencias ya no eran significativas, y los espacios de la línea media se habían cerrado en ambos grupos cuando los sujetos alcanzaron el promedio de 19 años de edad.

Popovich et al.(1977) (8) realizaron el seguimiento a 471 niños, con una edad promedio de 9 años de edad; luego de 7 años, cuando los niños habían alcanzado la edad de 16 años de edad, encontraron que en el 83.2\% de ellos el diastema interincisal había cerrado por completo, 7.4\% tenía un espacio menor de $0.5 \mathrm{~mm}$ y tan sólo en el 9.4\% el diastema era mayor de $0.5 \mathrm{~mm}$. Esta tendencia porcentual indica que el diastema se va cerrando paulatinamente durante el desarrollo normal del individuo.

Taylor (1939) (4) examinó en California (EE. UU.) un grupo de escolares entre 5 1⁄2 y 18 años de edad, y encontró que el diastema disminuía de $98 \%$ a la edad de 6 años, a $49 \%$ a los 11 años de edad y 7\% a los 12 y 18 años de edad.

Weyman (13), en 1967, relacionó la presencia de diastema maxilar de la línea media con los estadios de erupción dentaria de las piezas maxilares anteriores. Luego de examinar 751 niños británicos de 7 a 16 años de edad, llegó a las siguientes conclusiones: con los incisivos centrales erupcionados, el 56.8\% tenía un amplio diastema interincisivo; cuando los incisivos laterales estuvieron presentes esta cifra disminuyó a $38 \%$, y luego de la erupción de los caninos sólo el 7.4\% tenía dicho espacio. El $5.7 \%$ de pacientes de 14 a 16 años de edad presentaba algún espacio interincisivo y mostraba una marcada tendencia a reducir el espacio de la línea media con el desarrollo de la dentición.

Sin embargo, existen algunos factores que pueden predisponer a la presencia del DMI luego de la maduración de la oclusión. Los componentes congénito y hereditario son mencionados como aspectos de gran importancia en la persistencia de tales espacios (1,2,24,41). Graber (42) (1991) relaciona los diastemas originados por frenillos con fuertes tendencias de origen familiar. El factor étnico o racial es mencionado también como otro de los aspectos importantes en la presencia de diastema maxilar de la línea media $(2,41)$, ya que algunas razas tienen tendencia a desarrollar arcos dentarios más amplios en relación al tamaño dentario (16); la raza negra (27) y grupos mediterráneos (16) muestran una mayor prevalencia a la presencia de este espacio. Gardiner (1967) (2) menciona que ésta es una característica muy común en las poblaciones del este de África.

En 1973, Richardson et al. (27) realizaron un estudio en una población birracial de 5307 niños de 6 a 14 años de edad, en el cual la población negra mostró la prevalencia más alta del diastema maxilar de la línea media que la población blanca en casi todos los grupos etáreos, excepto a los 8 años de edad.

Es también importante mencionar que un marcado espacio persistente entre los incisivos centrales en la denti- ción permanente puede traer consigo problemas estéticos y de oclusión o alterar la pronunciación de ciertas sílabas (22), aunque la importancia estética puede variar dependiendo del grupo étnico o cultural (29).

\section{Etiología de diastemas patológicos}

Los factores etiológicos de los diastemas patológicos son complejos y diversos y por lo general se interrelacionan $(7,11)$.

Huang y Creath (16) en 1995 realizaron una interesante revisión de la literatura, señalando como principales factores etiológicos del DMI los siguientes :

- Hábitos perniciosos prolongados (succión de labio inferior, succión digital), los cuales pueden cambiar el equilibrio de las fuerzas peribucales, originando alteraciones dentofaciales.

- Desequilibrio muscular en la cavidad bucal, causado por macroglosia, linfangiomas, músculos flácidos y empuje lingual.

- Impedimento físico, dientes supernumerarios (mesiodents), frenillo labial superior anómalo, quistes, fibromas, cuerpos extraños e inflamación periodontal asociada.

- Anomalías en la estructura maxilar, por un crecimiento excesivo ya sea por factores endocrinos (alteración de la glándula pituitaria), hereditarios o congénitos que pueden originar espacios generalizados entre los dientes, por sutura abierta en forma de V o de pala, sutura media palatina idiopática como resultado de tratamiento ortopédico u ortodóntico; o pérdida de soporte óseo, debido a problemas sistémicos o periodontales.

- Anomalías dentales y maloclusiones, que incluyen alteraciones de tamaño, forma y posición de dientes adyacentes, dientes ausentes y patrones de oclusión anormales, como incisivos rotados, maloclusión clase II división I.

Otros autores señalan también como factores causales: mordida profunda, dientes inferiores de mayor tamaño, apiñados o rotados (24), respiración bucal, 
incisivos centrales anquilosados (43), mordida abierta (2), posibles trastornos neuromusculares (39), tratamientos de ortodoncia u ortopedia iatrogénicos (2) y colapso de mordida posterior (20).

\section{Diagnóstico del diastema patológico}

El diagnóstico de la normalidad o patología de un diastema no siempre es tan sencillo, de allí la necesidad de una correcta valoración fisiológica (30).

Frente a un niño con diastema central maxilar deberá realizarse una cuidadosa historia médico-odontológica a fin de investigar si las características de este espacio son normales para su edad y su paralelo crecimiento y desarrollo físico y dental $(5,44)$. Son también importantes la raza, los antecedentes congénitos (1) y familiares (22), presencia de alteraciones sistémicas que condicionen la existencia de dicho espacio, como por ejemplo, desequilibrios hormonales $(1,4)$ o alteraciones neuromusculares (39).

El examen clínico deberá evaluar inserción anómala del FLS (2, 16, 37), presencia de hábitos deletéreos $(5,16,30$, 43), alteración del equilibrio de la musculatura peribucal (9), problemas ortodónticos $(9,35,43)$, erupción dentaria (5) y sus alteraciones (9), trastornos en la forma, tamaño y número de las piezas dentarias (2, 15, 35), secuelas de traumatismos, caries dental (30) y pérdida ósea por problemas periodontales (21) o sistémicos $(4,16)$. La edad del paciente, según Fischer y Psaltis (5) (1981), es el factor clínico decisivo.

Los exámenes radiográficos son imprescindibles para el diagnóstico. Son necesarias radiografías panorámicas y periapicales del sector anterosuperior a fin de evaluar: edad dental del paciente, impedimentos físicos, agenesias, morfología sutural, anquílosis, problemas de erupción y alineamiento $(16,45,46)$.

En algunas ocasiones es necesario obtener registros ortodónticos completos, como modelos de estudio, fotografías clínicas y análisis de Bolton, si el caso así lo amerita (16).

Para un examen minucioso del diastema interincisal, Araujo y Bolognese (24) (1983) nos sugirieron los siguien- tes pasos :

a) Precisar si sólo existe el espacio de la línea media maxilar, o si existen espacios generalizados.

b) Comparar el tamaño de los dientes entre sí y/o con una tabla de medidas promedio.

c) Una buena radiografía de este sector con el rayo central perpendicular al septo interincisal.

A esto Oesterle y Shellhart (29) (1999) añaden :

d) Un minucioso examen periodontal de la zona.

Valladares-Neto et al. (11) (1996) señalan que los ejes axiales de las coronas de los incisivos centrales podrían sugerirnos la probable causa del diastema, así :

- Cuando las coronas son divergentes nos podrían hacer pensar en la presencia de un frenillo labial anómalo.

- Incisivos centrales con ejes paralelos o convergentes podrían estar vinculados a la presencia de mesiodents, agenesia o microdoncia de incisivos laterales, falta de coalescencia sutural, discrepancia de tamaño dentario u odontoma.

- Incisivos centrales vestibulizados se asocian a una maloclusión clase II, división I, labio hipotónico o hábitos bucales indeseables.

\section{Relación diastema-frenillo}

Por muchos años la relación diastema-frenillo se ha constituido en la preocupación de los investigadores del tema. ¿Es el frenillo anómalo el que da origen al diastema?, ¿o es la presencia de un espacio entre los incisivos centrales lo que posibilita la existencia de un frenillo amplio? Ese es el punto de discusión.

Según Vono et al. (22) (1973) y Baer y Benjamín (36)(1975), estas teorías contradictorias tendrían su base en la inseguridad por parte del clínico, en no saber diferenciar un verdadero frenillo anómalo de uno que no lo es, sobre todo en la infancia, donde existe una alta frecuencia de frenillos de inserción baja.

Gibbs (37) (1968) y Walter (47) (1978) nos recuerdan que la presencia de un diastema entre los incisivos centrales maxilares no siempre se debe a un frenillo de inserción baja, existen también otros factores etiológicos. Un frenillo deberá ser considerado anómalo sólo cuando todas las demás causas han sido eliminadas. ${ }^{5,35}$

La discusión acerca de la relación diastema-frenillo se puede agrupar en tres grandes posiciones $(3,11,48)$ :

1. Los que sostienen que el FLS de inserción baja o anómalo es el responsable del diastema: Dewey (49) (1918), Parker (50) (1937), Taylor (4) (1939), Dewel (35) (1946), Ceremello (9) (1953), Hernández (51) (1959), Ewen (52) (1968), Gibbs (37)(1968), Vono et al.(22) (1973), Fischer y Psaltis (5) (1981), Passani et al.(53) (1987), Albuqerque (54) (1990), Costa-Pinto y Gregori (55)(1975), Araujo y Bolognese (24) (1981); y a juicio de Curran (56) (1950) no sería el tejido en sí sino el movimiento de éste lo que produciría el distanciamiento interincisal.

2. Los que sostienen que el frenillo anómalo es resultado del diastema, en tanto que la presión ejercida por los dientes adyacentes es insuficiente para producir su atrofia. Sus principales sostenedores son : Tait (1) (1934), Baum (15) (1966), García (57) (1977), Compostella (30) (1980), Oesterle y Shellhart (29) (1999) y

3. Aquellos que sostienen que el frenillo anómalo y el diastema son entidades independientes una de la otra. Estudios realizados por Ceremello (9) (1953) y Vanzato et al. (12) (1999) habrían demostrado la independencia del FLS respecto del diastema interincisal.

Cabe resaltar que la mayoría de autores consultados se inclinan a sostener que el FLS anómalo puede ser un importante factor etiológico en casos de diastemas patológicos.

\section{Tratamiento del problema diastema- frenillo}

Son diversas las conductas terapéuticas recomendadas por los estudiosos frente al problema diastema-frenillo, no solo respecto al momento oportuno para 
su realización, sino también en lo referente a la diversidad de técnicas existentes. ${ }^{11}$

Para la solución del problema diastema-frenillo, la literatura nos presenta tres procedimientos básicos :

a. Procedimiento ortodóntico, que utiliza fuerzas ortodónticas que comprimen el tejido hipertrófico, promoviendo la atrofia y migración de la inserción gingival del FLS, lo que hace innecesaria la frenectomía (37).

b. Procedimiento quirúrgico. Según Edwards (7) (1977) pueden ser : frenectomía (remoción completa del frenillo, incluida su inserción en el hueso) y frenotomía (reposición de la inserción del frenillo hacia apical) muy utilizada con fines periodontales. Otros procedimientos, mucho más radicales han sido también propuestos, tales como osteotomía subapical, corticotomías, septotomias, etc. (16).

c. Procedimiento ortodóntico-quirúrgico, que consiste en que luego de realizar la aproximación de los incisivos centrales, mediante aparatología ortodóntica, se realiza la cirugía del frenillo fibroso, a fin de evitar la formación de una amplia masa de tejido cicatrizal, que impediría la aproximación de los incisivos. Esto, seguido de un periodo de estabilización para evitar recidivas (30).

Así mismo la recesión quirúrgica del frenillo debería realizarse por las siguientes razones :

- Funcionales, cuando interfieran con las funciones de alimentación y fonación $(26,44,51,58)$.

- Estéticas, según Jacobs (59) (1932), cuando causen o mantengan una eversión o inversión del labio superior.

- Ortodónticas, como parte del tratamiento ortodóntico en el cierre de diastemas (58).

- Protésicas, cuando impidan la retención y adaptación de prótesis en pacientes edéntulos (58).

- Periodontales, cuando dificulten el correcto cepillado, permitiendo acumulación de placa y predispongan a enfermedad periodontal $(58,60)$.
Anderman (61) (1984) y Pick y Colvard (62) (1993) recomiendan algunas técnicas quirúrgica incruentas, por las ventajas que presentan. Así tenemos:

- La electrocirugía, que permite una mejor visibilidad de la zona, el corte del tejido es fácil y rápido; por ello se dice que es un procedimiento aceptado por los niños (61).

- El láser $\mathrm{CO}_{2}$ (dióxido de carbono) y Nd: YAG (neodimio: itrio-aluminio-granade), que tiene efectos hemostáticos, acción antiedematosa, escaso dolor post-quirúrgico y mínima formación de tejido cicatrizal (62).

Por mucho tiempo la edad recomendada para iniciar el tratamiento ha sido motivo de controversia y aún continúa siendo discutible. Por ello la edad dentaría del individuo sería la mejor referencia práctica para emprender el tratamiento, según Valladares Neto et al (11). (1996).

$\mathrm{Al}$ evaluarse la literatura, se puede observar una diversidad de preferencias de los autores con respecto al inicio del tratamiento :

- Durante el primer año de vida, cuando el frenillo es de tipo tectolabial persistente, adiposo, fibroso y se une al labio impidiendo la alimentación y fonación del niño. Comparten esta opinión Curran (56) (1950), Walter et al. (26) (1997) y Pereira (44) (2001).

- Durante el proceso de exfoliación de los incisivos deciduos y la erupción de los permanentes, es decir, alrededor de los 6 a 8 años de edad, criterio sostenido por Walter et al. (26) (1997).

- Después de la erupción de los incisivos permanentes, opinión sostenida por Parker (50) (1937) y Fonseca (58) (1970).

- Cuando los caninos permanentes se encuentran en proceso de erupción o después de la erupción de éstos, mayoritariamente sostenido por Jacobs (59) (1932), Shirazy (63) (1938), Taylor (4) (1939), Curran (56) (1950), Gibbs (37) (1968), Otero (64) (1973), Graber (42) (1991), Passani et al. (53) (1987), Kilgore (65) (1987), Verluyten (6) (1989), Kabam (31) (1990), Wilson y Montgomery (66) (1991).

- Después de la erupción de las segundas molares permanentes, momento preferido por Ceremello (9) (1953), Dewel (17) (1966), Ewen (52) (1968), Baer y Benjamin (36) (1975), Compostella (30) (1980).

Se puede señalar que la mayoría de los autores recomiendan esperar el momento de la erupción de los incisivos y caninos permanentes superiores para iniciar algún tratamiento. Sólo cuando el crecimiento normal y desarrollo de los procesos maxilares y la oclusión hayan fracasado o cuando sea evidente la presencia del frenillo anómalo, tipo "asa delta” o "pala de ventilador” (67), tan perjudicial para el paciente, la terapia interceptiva y oportuna será necesaria. El amplio conocimiento que se tenga del tema, la experiencia del clínico y el análisis cuidadoso de cada caso siempre ayudarán a determinar el momento oportuno para su tratamiento.

La elección de los procedimientos o técnicas más adecuadas siempre será decisión del clínico y resultará ventajoso para el paciente, si ello responde a un trabajo multidisciplinario donde intervengan diferentes especialistas vinculados al problema : odontopediatra, ortodoncista, periodoncista, cirujano y fonoaudiólogo, entre otros.

\section{Referencias bibliográficas}

1. Tait $\mathrm{CH}$. The median frenum of the upper lip and its influence on the spacing on the upper central incisor teeth. Dental Cosmos 1934; 76(2):991-992.

2. Gardiner JH. Midline spaces. Dent Pract 1967; 17(8):287-298.

3. Ferguson MWJ. Pathogenesis of abnormal midline spacing of human central incisors. Br Dent J 1983; 154:212-218.

4. Taylor JE. Clinical observations relating to the normal and abnormal frenum labii superioris. Am J Orthod 1939; 25:646-60.

5. Fischer TJ, Psaltis GL. The diastema and the abnormal frenum. J Dent 
Child 1981; 4:264-268.

6. Verluyten P. Maxillary central diastema. Rev Belge Med Dent 1989; 44(3):117-22.

7. Edwards JG. The diastema, the frenum, the frenectomy: a clinical study. Am J Orthod 1977; 71(5):489-508.

8. Popovich F, Thompson GW, Main PA. The maxillary interincisal diastema and its relationship to the superior labial frenum and intermaxillary suture. Angle Orthod 1977; 47(4):265-271.

9. Ceremello PJ. The superior labial frenum and the midline diastema and their relation to growth and development of the oral structures. Am J Orthod 1953; 39(2):120-139.

10. Madeira AA, Minatti EJ. Observações clínicas e radiográficas, relacionando o diastema mediano, com o freio maxilar mediano. Rev Gaucha Odont 1979; 27(4):226-230.

11. Valladares-Neto JV, Ribeiro AV, Silva Filho OG. O dilema do diastema mediano e o freio labial superior: análise de pontos fundamentais. ROBRAC 1996; 6(19):9-17.

12. Vanzato J, Sampaio JEC, Correa de Toledo BE. Prevalência do freio labial anômalo e diastema mediano dos maxilares e sua interrelação. Rev Gaucha Odont 1999; 47(1):27-34.

13. Weyman J. The incidence of median diastemata during the eruption of the permanent teeth. Dent Practit 1967; 17(8):276-278.

14. Minatti EJ, Madeira AA. Prevalência do diastema superior en crianças melanodermas. Rev Catarinense Odont 1981; 8(2):22-25.

15. Baum AT. The midline diastema. J Oral Med 1966; 21 (1):30-39.

16. Huang W J, Creath CJ. The midline diastema : a review of its etiology and treatment. Pediatr Dent 1995; 17(3):171-179.

17. Dewel BF. The labial frenum, midline diastema, and palatine papilla: a clinical analisys. Dent Clin North Am 1966; 10(3):175-184.

18. Higley LB. Maxillary labial frenum and midline diastema. J Dent Child 1969; 36(6):413-414.

19. Bergström K, Jensen R, Martensson
B. The effect of superior labial frenectomy in cases with midline diastema. Am J Orthod 1973; 63(6):633638.

20. Campbell PM, Moore JW, Matthews JL. Orthodontically corrected midline diastemas. a histologic study and surgical procedure. Am J Orthod 1975; 67(2):139-158.

21. Herremans EL. Anterior diastema: frenectomy. Dent Surv 1971; 47(2):33-37.

22. Vono BG, Freitas MI, Tarquino BR. Uma pesquisa sobre diastema incisal-freio labial. Rev Brasileira Odont 1973; 30:138-141.

23. Vono BG, Freitas MI, Tarquino BR. ¿Hay relaciones íntimas entre diastema y frenillo?. Fauchard 1970; 10:422-424.

24. Araújo LG, Bolognese AM. Diastema Interincisal $\mathrm{x}$ freio labial anormal. Rev Brasileira Odont 1983; 40(5):20-28.

25. Vera VR. Maloclusiones, gingivitis, frenillo labial superior y diastemas en escolares de Itá (Paraguay). Rev Odont Circ Odont Parag 1974; 20(2):79-87.

26. Walter LRF, Ferelle A, Issão M. Odontología para o bebê. Sao Paulo: Artes Médicas, 1997.

27. Richardson ER, Malhotra SK, Henry M, Little RG, Coleman HT. Biracial study of the maxillary midline diastema. Angle Orthod 1973; 43 (4):438-443.

28. Sullivan TC, Turpin DL, Artun J. A postretention study of patients presenting with a maxillary median diastema. Angle Orthod 1996; 66(2):131-138.

29. Oesterle LJ, Shellhart WC. Maxillary midline diastemas: a look al the causes. J Am Dent Assoc 1999; 130:85-94.

30. Compostella EA. Enfoque multidisciplinario de los diastemas y los frenillos bucales. Rev Asoc Odont Argent 1980; 68(2):74-80.

31. Kabam LB. Cirugía bucal y maxilofacial en niños. México: Ed. Interamericana, 1990.

32. Baume LJ. Physiological tooth migration and its significance for the development of occlusion. J Dent Res 1950; 29(2):123-131, 338-348.

33. Mc Donald RE. Odontología pediátrica y del adolescente. 6ta Ed. Madrid: Editorial Mosby-Doyma, 1995.

34. Mc Callum CHA. Cirugía bucal para niños. En: Finn SB. Odontología pediátrica. 4ta. Ed. México: Editorial Interamericana, 1976:365-367.

35. Dewel BF. The normal and the abnormal labial frenum : clinical differentiation. J Am Dent Assoc 1946; 33(3):318-329.

36. Baer PN, Benjamín SD. Enfermedad periodontal en niños y adolescentes. Buenos Aires: Edit Mundi, 1975.

37. Gibbs SL. The superior labial frenum and its orthodontic considerations. NY State Dent J 1968; 34:550-553.

38. Broadbent BH. Ontogenic development of occlusion. Angle Orthod 1941; XI(4):223-241.

39. Rivero JC, Martínez-Gonzales JM, Peris RMP, Del Canto MC. Frenulectomías con fotobisturí láser $\mathrm{CO}_{2}$ en la práctica ortodóncica. Rev Actual Odontoestomatog Española 1993; LIII(422):27-33.

40. Henry SW, Levin MP, Tsaknis PJ. Histologic features of the superior labial frenum. J Periodontol 1976; 47(1):25-28.

41. Kopel H, et al. The labial frenum. J Dent Child 1955; 22(3):141-143.

42. Graber TM. Ortodoncia. 3ra Ed. México: Editorial Interamericana, 1991:892.

43. Bedell WR. Nonsurgical reduction of the labial frenum with and without orthodontic treatment. J Am Dent Assoc 1951; 42:510-515.

44. Pereira MBB. Urgências e emergências en odontopediatría. Curitiba: Ed. Maio, 2001.

45. Marzola C. Cirugías pre-protéticas. Rev Brasileira Odont 25(154):325333, 1968.

46. Walter LRF. Prevalência dos diferentes tipos de freio labial em escolares londrinenses. Rev Ass Paul Cirurg Dent 1980; 34(5):426-430.

47. Walter LRF. Estudo da prevalência dos diferentes tipos de freio labial superior em escolares da cidade de 
Londrina: (Escolas Municipais Periféricas) [Tesis Libre Docente]. Londrina: Universidad Estadual de Londrina, 1978.

48. Galassi MAS, Toledo BEC, Sampaio JEC. A importância do freio labial nas estructuras periodontais. Rev Gaucha Odont 1994; 42 (1):12-14.

49. Dewey M. Three types of frenum labiorum. Orthod Int J 1918; IV:461462.

50. Parker, DB. Surgical consideration of abnormal frena. Inter J Orthod Oral Surg 1937; 23:1141-1148.

51. Hernández L. Diastema de los incisivos centrales superiores y frenectomía labial [Tesis Bachiller]. Lima : Universidad Nacional Mayor de San Marcos, 1959.

52. Ewen SJ. Frena : their roles especially in periodontics. NY State Dent J 1968; 34:626-630.

53. Passani CA, Santos Pinto CA, et al. Tratamento do freio labial hipertrófico. Rev Odont Clin 1987; 1(2):10-12.

54. Albuqerque MES. Estudo da topografia e da morfologia do freio labial superior de crianças de 0 a 36 meses [Tesis Maestría]. São Paulo : Universidade do São Paulo, 1990.

55. Costa Pinto ML, Gregori C. Aspectos embriológico, anatômico, fisiológico e cirúrgico, relacionados com freio labial. Rev Ass Paul Cirurg Dent 1975; 29(3):15-31.

56. Curran M. Superior labial frenotomy J Am Dent Assoc 1950; 41(4):419422.

57. García MO. Freio maxilar. Rev Quintessence 1977 ; 4(7):55-58.

58. Fonseca GD. Frenectomia labial: presentación de una nueva técnica. Rev Fed Odont Ecuat 1970; 1(3):307311.

59. Jacobs MH. The abnormal frenum labii. Dental Cosmos 1932; 74(5):436439.

60. Gottsegen R. Frenum position and vestibule depth in relation to gingival health. Oral Surg 1954; 10:10691078.

61. Anderman LL. Pediatric dental electrosurgery. NY State Dent J June/ July:348-350, 1984.

62. Pick RM, Colvard MD. Current status of lasers in soft tissue dental surgery. J Periodontol 64(7):589602, 1993.

63. Shirazy E : The frenum labii superioris. JADA \& Dent Cosmos 1938; 25:761-762.

64. Otero J. Frenillo superior anormal. Rev Cub Est 1973; 10:131-135.

65. Kilgore TB. Cirugía bucal y protocolo odontológico hospitalario. En: Braham RL, Morris ME. Odontología pediátrica. Argentina : Ed. Médica Panamericana, 1987. p. 350-351.

66. Wilson S, Montgomery D. Anestesia local y cirugía bucal en niños. En:Pinkham JR. Odontología pediátrica. 1ra Ed. México: Edit Interamericana, 1991:339.

67. Seibt GB, Regio RAS, Chiapinotto G. Frenectomia. Rev Gaucha Odont 1994; 42(4):215-218.

\section{Dirección de Correspondencia}

María Elena Díaz Pizán

Verrochio 345 Lima 41- Perú

540-0362

ciepde@amauta.rcp.net.pe 